\title{
Tibiofibular Synostosis
}

\author{
N. K. Sferopoulos* \\ Department of Orthopaedic Surgery, 'G. Gennimatas' Hospital, 54635 Thessaloniki, Greece
}

*Corresponding Author: N. K. Sferopoulos, Department of Orthopaedic Surgery, 'G. Gennimatas' Hospital, 54635 Thessaloniki,Greece,E-mail: sferopoulos@in.gr,sferopoulos@yahoo.com

\begin{abstract}
Synostosis of any of the three joints that the tibia and fibula share is extremely rare. Tibiofibular synostosis may occur in skeletally immature patients, adolescents and adults. It may be congenital, idiopathic (primary) or acquired (secondary). Congenital lesions are usually localized in the proximal or distal tibiofibular joint and caused by the coalescence of 'kissing' osteochondromata in patients suffering from multiple hereditary exostoses. Tibiofibular synostosis with no associated syndromes or deformities may be either idiopathic or acquired. Acquired cases are usually post-traumatic, secondary to fractures and injuries, or iatrogenic, complicating orthopaedic interventions such as fracture fixation, osteotomies or grafting .
\end{abstract}

Keywords: Tibiofibular synostosis, congenital, idiopathic, acquired

\section{EDITORIAL}

The lesion may appear as proximal, middle (diaphyseal) or distal tibiofibular synostosis. Despite its localization, synostosis appearing as a congenital lesion or occuring before the closure of the physeal plates is usually complicated by shortening of the lateral malleolus, ankle valgus and prominence of the fibular head at the knee. These lesions may potentially lead to ankle pain, since the synostosis interferes with the normal motion that occurs between the tibia and fibula during weight-bearing [1].

Synostosis of the proximal tibiofibular joint has been reported in both children and adults. Predisposing factors may include the syndrome of multiple hereditary exostoses, knee valgus and other generalized syndromes, like the 49, XXXXY karyotype. The absence of any growth abnormalities indicates that the synostosis occurred after physeal closure. Synostosis of the proximal tibiofibular joint is very rare in adults. It is rarely associated with complaints and may, therefore, be easily missed. It is usually primary but it may also be iatrogenic. The latter has been reported secondary to surgical reconstruction and grafting of a depressed fracture of the lateral tibial plateau as well as following proximal tibial osteotomies, due to relative instability during bony healing. No evidence that the synostosis accelerated the onset or progression of degenerative changes to the ipsilateral knee has been documented [2-18].

Synostosis of the middle tibiofibular joint (formed by the interosseous membrane), also referred as diaphyseal tibiofibular synostosis, may be localized either between the proximal and middle third or between the middle and distal third of the tibia. Pathogenesis may implicate repeated trauma (stress injuries) or severe injury causing either bleeding or subperiosteal dissection across the interosseous membrane, causing new bone formation, which bridges over the tibia and fibula. Although most patients are asymptomatic, some may experience disabling pain and discomfort during exercise. Diaphyseal tibiofibular synostosis has been described as a rare cause of anterior compartment pain or ankle pain related to sport activities. Increased intramuscular pressure due to bleeding from repeated microfractures of the synostosis might explain the symptoms. They could also be explained by alteration of the downward and medial movement of the fibula during gait by the foot flexors. Incidental radiographic detection of tibiofibular synostosis is more frequent in professional football players than in the normal population. Magnetic resonance imaging with paramagnetic contrast is recommended, to exclude a primary bony tumor, especially in 
pediatric cases, and to determine the relation of the synostosis to the neurovascular structures.

Treatment should always start with conservative measures, such as rest, ice compression, nonsteroidal anti-inflammatory drugs and ultrasound-guided steroid injection. Surgery should be reserved for patients not improving after conservative treatment. Management and optimal treatment of tibiofibular synostosis in athletes remain controversial. Conservative treatment is recommended initially. Surgical excision should be reserved for the athletically active patient whose symptoms are gradually progressing and disabling, and only after the synostosis appears as mature cortical bone on radiographs. Idiopathic symptomatic diaphyseal tibiofibular synostosis usually shows poor response to conservative treatment and should be treated by surgical resection. A high risk of recurrence after surgical treatment has been reported, so meticulous attention should be given to hemostasis during surgery. A fractured synostosis and a recurrent compartment syndrome after a fractured synostosis have been reported as rare complications of the lesion [1928].

The distal tibiofibular joint is a syndesmosis formed by two bones and four ligaments. The distal tibia and fibula, which form the osseous part, are linked by the distal anterior tibiofibular ligament, the distal posterior tibiofibular ligament, the transverse ligament and the interosseous ligament. The distal tibiofibular joint begins at the level of origin of the tibiofibular ligaments from the tibia and ends where these ligaments insert into the fibular malleolus. The distal fibula has a $2.4 \mathrm{~mm}$ extorsion under the condition of weight bearing. This serves to deepen the ankle mortise and tighten the interosseous membrane, which results in increased stabilization of the ankle. Therefore, synostosis between the tibia and fibula will be associated with a restricted fibular rotation and translation and accordingly may be complicated by a painful decreased ankle motion, usually during weight bearing and push off. Synostosis of the distal tibiofibular joint is usually acquired but it may also be idiopathic or congenital. The latter has been noted in patients suffering from the syndrome of multiple hereditary exostoses, fibrodysplasia ossificans progressive, flourosis, scurvy and hemophilia. The history of prior ankle trauma or surgically treated ankle fractures associated with ossification arising from the inner aspects of both the distal tibia and fibula is typical of post-traumatic tibiofibular synostosis and helps to the differentiation of this benign lesion from the rare surface osteosarcoma. Pathogenetic mechanisms include either primary traumatic laceration of the distal interosseous membrane or iatrogenic damage of the interosseous membrane caused by intra-operative soft tissue damage, periosteal stripping, deep drilling and damage by Kirschner wires or screws. Fixation of the distal interosseous membrane by cancellous screws may induce synostosis, while in time removal of the screw is necessary to avoid synostosis of the distal tibiofibular joint.

The radiographic extent of calcification or synostosis at the level of the distal interosseous membrane or syndesmosis on the plain radiographs has been defined as: no or minor calcifications, severe calcification, and complete synostosis. Syndesmotic screw fixation, male sex, tibiotalar dislocation and Weber type-C ankle fractures are significant risk factors in the formation of post-operative incomplete bony bridging or complete distal tibiofibular synostosis. Despite relatively extensive imaging findings of incomplete or complete bony bridging, functional outcomes are usually minimally affected and specific treatment is usually not recommended. A fibular stress fracture proximal to the superior border of the synostosis is the only reported complication in an athlete with distal tibiofibular synostosis.

Two patterns of synostosis after the treatment of pediatric distal tibiofibular fractures are encountered: focal and extensive. The focal type is more prevalent than the extensive type, which is more likely to occur due to high-energy trauma. When a tibiofibular cross-union develops, it should be closely followed, since it may be complicated by growth abnormalities leading to limb length discrepancy, valgus malalignment of the ankle joint, and gait or biomechanical abnormalities, which may require surgical removal of the synostosis. [29-60]. 


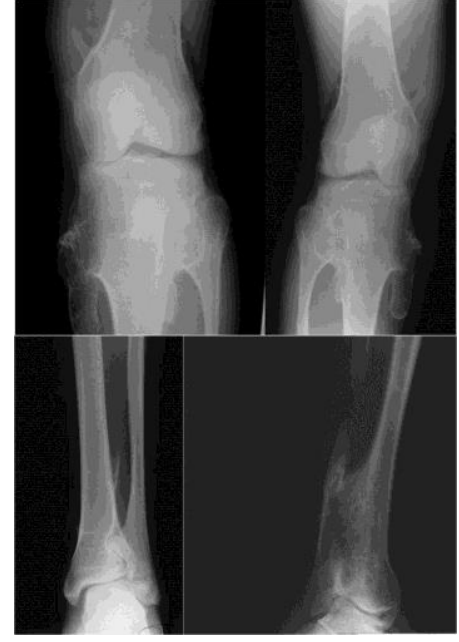

Figure1: A 21-year-old man suffering from the syndrome ofmultiple hereditary exostoses. A bilateral complete synostosis was detected in the proximal tibiofibular joints most likely due to facing osteochondromata of both bones that showed an interlocking growth at abutting parts. Synostosis of the right distal tibiofibular joint was caused by the coalescence of 'kissing' osteochondromata. A growing osteochondroma arising from the fibula caused erosion in the contagious surface of the neighbouring tibia on the left distal tibiofibular joint

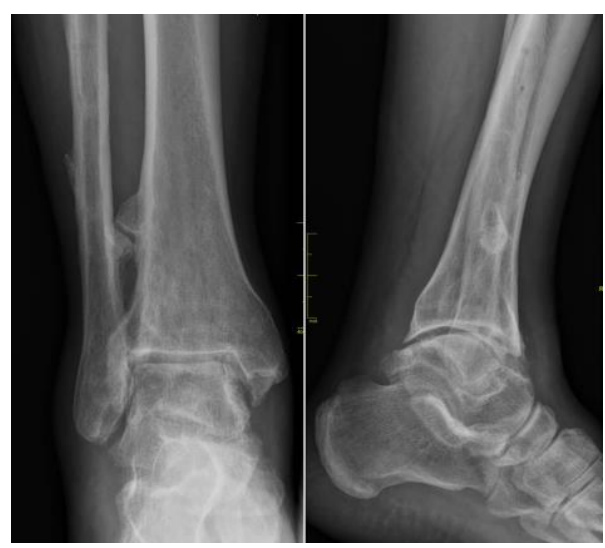

Figure2: Radiographs of a 38-year-old man who was surgically treated for a trimalleolar fracture 20 years ago

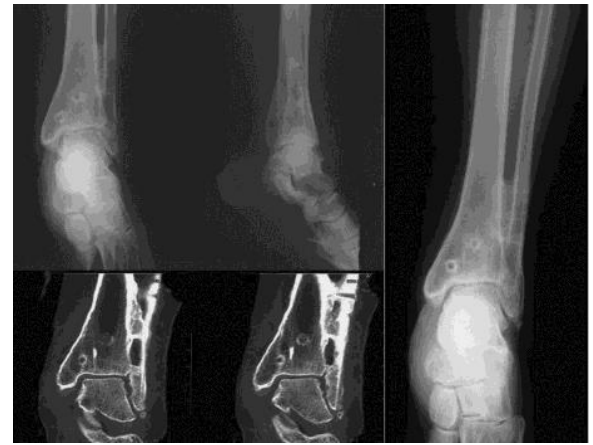

Figure3: A 42-year-old woman who was surgically treated for a trimalleolar fracture 6 years ago. Radiographs and computed tomography images 3 months after implant removal (left) and anteroposterior radiograph at 8 months afterimplant removal (right).

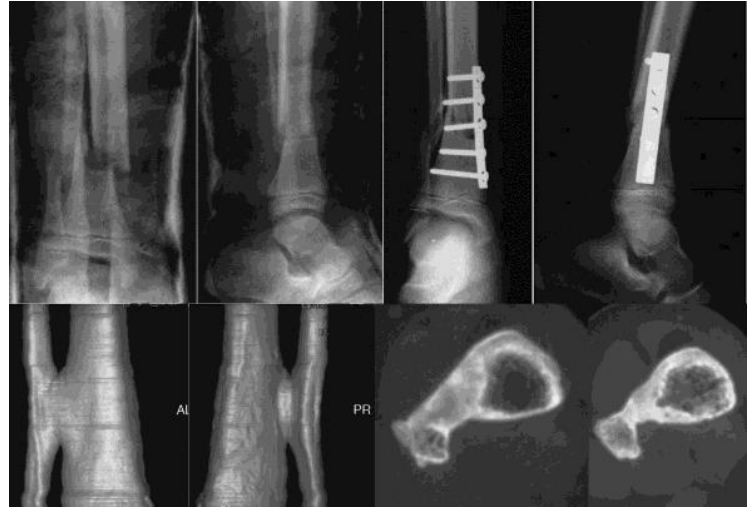

Figure4: A 10-year-old boy was admitted after being hit by a motorbike. The fractured tibia and fibula healed after a plate and screw fixation of the tibia. $3 D$ computed tomography reconstruction, and axial images showed diaphyseal tibiofibular synostosis.

\section{REFERENCES}

[1] Frick SL, Shoemaker S, Mubarak SJ. Altered fibular growth patterns after tibiofibular synostosis in children. J Bone Joint Surg Am. 2001; 83(2): 247-54. PMID: 11216687.

[2] Ogden JA. The anatomy and function of the proximal tibiofibular joint. Clin Orthop Relat Res. 1974; 101: 186-91. PMID: 4837930.

[3] Von Törne O. Tibiofibular synostoses. Z Orthop Ihre Grenzgeb. 1977; 115(3): 372-375. PMID: 888525 .

[4] Wong K, Weiner DS. Proximal tibiofibular synostosis. Clin Orthop Relat Res. 1978; 135: 45-7. PMID: 709951.

[5] Eichenblat $M$, Nathan $H$. The proximal tibio fibular joint. An anatomical study with clinical and pathological considerations. Int Orthop. 1983; 7(1): 31-9. PMID: 6618736.

[6] Gamble JG. Proximal tibiofibular synostosis. J Pediatr Orthop. 1984; 4(2): 243-5. PMID: 6699 166.

[7] O'Dwyer KJ. Proximal tibio-fibular synostosis. A rare congenital anomaly. Acta Orthop Belg. 1991; 57(2): 204-8. PMID: 1872166.

[8] Bessler W, Eich G, Stuckmann G, Zollikofer C. Kissing osteochondromata leading to synostoses. Eur Radiol. 1997; 7(4): 480-5. PM ID: 9204324 . DOI: $10.1007 / \mathrm{s} 003300050188$.

[9] Takai S, Yoshino N, Hirasawa Y. Unusual proximal tibiofibular synostosis. Int Orthop. 1999; 23(6): 363-5. PMID: 10741527. PMCID: PMC3619841.

[10] Nishikawa T, Yamamoto T, Yoshiya S, Kurosaka M. Intermittent peroneal mononeuropathy due to proximal tibiofibular synostosis. J Neurol. 2003; 250(3): 352-3. PMID: 12749315.

[11] Bozkurt M, Doğan M, Turanli S. Osteochondroma leading to proximal tibiofibular synostosis as a cause of persistent 
ankle pain and lateral knee pain: a case report. Knee Surg Sports Traumatol Arthrosc. 2004; 12(2): 152-4. PMID: 12942232. DOI: 10.10 07/s00167-003-0375-6.

[12] Mandal S, Coleman N. Proximal tibiofibular synostosis following tibial nailing: a case report and review of literature. Injury Extra 2005; 36(10): 418-420.

[13] Lenin Babu V, Shenbaga N, Komarasamy B, Paul A. Proximal tibiofibular synostosis as a source of ankle pain: a case report. Iowa Orthop J. 2006; 26: 127-9. PMID: 16789462. PMCID: PMC1888597.

[14] Nishimura T, Nii E, Urawa M, Nishiyama M, Taki S, Uchida A. Proximal tibiofibular synostosis with 49XXXXY syndrom, a rare congenital bone anomaly. J Orthop Sci. 2008; 13(4): 390-5. PMID: 18696202. DOI: 10.100 7/s00776-008-1230-x.

[15] Sferopoulos NK. Synostosis of the proximal tibiofibular joint. Case Rep Med. 2010; 2010: 794594. PMID: 20592991. PMCID: PMC289 2697. DOI: $10.1155 / 2010 / 794594$.

[16] Van Ooij B, van Ooij A, Morrenhof JW, van Dijk CN. Proximal tibiofibular synostosis as a possible cause of a pseudoradicular syndrome: a case report. Knee Surg Sports Traumatol Arthrosc. 2011; 19(12): 2115-8. PMID: 2122 2100. PMCID: PMC3217150. DOI: 10.10 07/s00167-010-1379-7.

[17] Wakayama T, Imanishi J, Yazawa Y, Okubo T, Kaneko K. Bilateral non-osteochondromarelated proximal tibiofibular synostosis. Skeletal Radiol. 2014; 43(12): 1737-42. PMID: 24915740. DOI: 10.1007/s00256-014-1932-4.

[18] Kaptan AY, Atik OŞ. Proximal tibiofibular synostosis. Eklem Hastalik Cerrahisi. 2017; 28(3): 207-9. PMID: 29125821. DOI: 10.5606/ehc.2017.00076.

[19] Flandry F, Sanders RA. Tibiofibular synostosis: an unusual cause of shin splint-like pain. Am J Sports Med. 1987; 15(3): 280-4. PMID: 3618880. DOI: $10.1177 / 036354658701500318$.

[20] Henry JH, Andersen AJ, Cothren CC. Tibio fibular synostosis in professional basketball players. Am J Sports Med. 1993; 21(4): 619-22. PMID: 8368426. DOI: 10.1177/036354659302 100423 .

[21] Magnusson HI, Westlin NE, Nyqvist F, Gärdsell P, Seeman E, Karlsson MK. Abnormally decreased regional bone density in athletes with medial tibial stress syndrome. Am J Sports Med. 2001; 29(6): 712-5. PMID: 11734482. DOI: 10.1177/03635465010290060 701.

[22] Fu JH, Hwang CC, Chao TH. Tibiofibular synostosis in a military soldier. J Med Sci 2003;
23(2): 135-138. http://jms.ndmcts gh.edu.tw/230 2135.pdf

[23] Horst F, Nunley JA. Tibiofibular synostosis stress fracture: a case report. Foot Ankle Int. 2004; 25(7): 507-9. PMID: 15319110. DOI: 10.1177/107110070402500711.

[24] Hanypsiak B, Bergfeld JA, Miniaci A, Joyce MJ. Recurrent compartment syndrome after fracture of a tibiofibular synostosis in a National Football League player. Am J Sports Med. 2007; 35(1): 127-30. PMID: $17130245 . \quad$ DOI: 10.1177/0363546506295698.

[25] James SH, Carpenter EC, Fairclough JA. Tibiofibular synostosis in a professional football player. J Bone Joint Surg Br. 2007; 89(1): 10911. PMID: 17259427. DOI: 10.13 02/0301620X.89B1.17945.

[26] Kobayashi S, Miyazaki T, Takeno K, Arakawa A. Diaphyseal tibiofibular synostosis in a runner. J Foot Ankle Surg. 2013; 52(5): 638-42. PMID: 23731941. DOI: 10.1053/j.jfas.2013.04. 001.

[27] Santa Maria DL, Shaw T, Allen M, Marin J. Fractured diaphyseal tibiofibular synostosis in an adolescent soccer player. PM R. 2015; 7(1): 84-7. PMID: 25171880. DOI: 10.1016/j.pmrj. 2014.08.947.

[28] Sonnery-Cottet B, Alessio-Mazzola M, Luz BF, Barbosa NC, Tuteja S, Kajetanek C, Dellal A, Thaunat M. Diaphyseal tibiofibular synostos is in professional athletes: Report of 2 cases. Orthop Traumatol Surg Res. 2016; 102(1): 135-8. PMID: 26615768. DOI: 10.1016/j.otsr.2015. 10.002 .

[29] Grobelski M. Congenital tibiofibular synostosis of the distal end of the lower leg. Arch Orthop Unfallchir. 1965; 57: 190-3. PMID: 14305987.

[30] McMaster J, Scranton, P. Tibiofibular synostosis, a cause of ankle disability. ClinOr thop.1975; 111:172-174.

[31] Whiteside LA, Reynolds FC, Ellsasser JC. Tibiofibular synostosis and recurrent ankle sprains in high performance athletes. Am J Sports Med 1978; 6: 204-208.

[32] Kärrholm J, Hansson LI, Selvik G. Changes in tibiofibular relationships due to growth disturbances after ankle fractures in children. $\mathbf{J}$ Bone Joint Surg Am. 1984; 66(8): 1198-1210. PMID: 6548476.

[33] Ney R, Jend JJ, Schöntag H. Tibiofibular mobility and arthrosis in patients with postoperative ossification in the area of syndesmosis of the upper ankle joint. Unfallchirurgie. 1987; 13(5): 274-277. PMID: 3122395.

[34] Harborne DJ, Lennox WM. Distal tibiofibular synostosis due to direct trauma. Injury. 1989; 20(6): 377-8. PMID: 2628342. 
[35] Vitale TD, Fallat LM. Distal tibiofibular synos tosis and late sequelae of an ankle sprain. $\mathrm{J}$ Foot Surg. 1990; 29(1): 33-6. PMID: 2319099.

[36] Taylor DC, Englehardt DL, Bassett FH 3rd. Syndesmosis sprains of the ankle. The influence of heterotopic ossification. Am J Sports Med. 1992; 20(2): 146-150. PMID: 1558241. DOI: $10.1177 / 036354659202000209$.

[37] Kottmeier SA, Hanks GA, Kalenak A. Fibular stress fracture associated with distal tibiofibular synostosis in an athlete. A case report and literature review. Clin Orthop Relat Res. 1992; 281: 195-8. PMID: 1499209.

[38] Böstman OM. Distal tibiofibular synostosis after malleolar fractures treated using absorbable implants. Foot Ankle. 1993; 14(1): 38-43. PMID: 8381109.

[39] Veltri DM, Pagnani MJ, O'Brien SJ, Warren RF, Ryan MD, Barnes RP. Symptomatic os sification of the tibiofibular syndesmosis in professional football players: a sequela of the syndesmotic ankle sprain. Foot Ankle Int. 1995; 16(5): $285-$ 290. PMID: $7633585 . \quad$ DOI: 10.1177/107110079501600507.

[40] Albers GH, de Kort AF, Middendorf PR, van Dijk CN. Distal tibiofibular synostosis after ankle fracture. A 14-year follow-up study. J Bone Joint Surg Br. 1996; 78(2): 250-2. PMID: 8666636.

[41] Kennedy MA, Sama AE, Sigman M. Tibiofibular syndesmosis and ossification. Case report: sequelae of ankle sprain in an adolescent football player. J Emerg Med. 2000; 18(2): 233240. PMID: 10699529.

[42] Cirlincione AS. Anatomical considerations of the ankle. Clin Podiatr Med Surg 2001; 18(3): 381-393. PMID: 11499169.

[43] Munjal K, Kishan S, Sabharwal S. Posttraumatic pediatric distal tibiofibular synostosis: a case report. Foot Ankle Int. 2004; 25(6): 429-33. PMID: 15215031. DOI: $10.11 \quad 77 / 10711$ 0070402500613.

[44] Dudkiewicz I, Ganel A, Blankstein A. Posttraumatic tibio-fibula synostosis in adults. Aktuelle Traumatologie. 2005; 35(4): 230-233.

[45] Bai XD, Xing GY, Yang CD, Ye QB. Operative treatment for separation of distal tibiofibular syndesmosis. Chin J Traumatol. 2006; 9(3): 175180. PMID: 16723076.

[46] Van den Bekerom MP, Lamme B, Hogervorst $\mathrm{M}$, Bolhuis HW. Which ankle fractures require syndesmotic stabilization? J Foot Ankle Surg. 2007; 46(6): 456-463. PMID: 17980843. DOI: 10.1053/j.jfas.2007.08.009.

[47] Hou ZH, Ye H, Shi JG, Zheng LB, Yao J, Ni ZM. Influence of distal tibiofibular synostosis on ankle function. Chinese Journal of
Traumatology 2009; 12(2):104-106. DOI: 10.3760/cma.j.issn.1008-1275.2009.02.008.

[48] Anas IY, Esomonu UG, Dimitrov ND, Rabiu IF, Saleh MS. Postraumatic tibiofibula synostosis of the distal 1/3 of the leg: A case study. Bayero Journal of Pure and Applied Sciences. 2009; 2(2): 31-33.

[49] Wani IH, Sharma S, Malik FH, Singh M, Shiekh I, Salaria AQ. Distal tibial interosseous osteochondroma with impending fracture of fibula - a case report and review of literature. Cases J. 2009; 2(1): 115. PMID: 19187551. PMCID: PMC2646691. DOI: 10.1186/17571626-2-115.

[50] Lee JY, Nam KY, Song KC. Distal tibiofibular synostosis after open reduction and internal fixation in a military soldier (A case report). Korean Foot Ankle Soc. 2010; 14(1): 105-107.

[51] Hermans JJ, Beumer A, de Jong TA, Kleinrensink GJ. Anatomy of the distal tibiofibular syndesmosis in adults: a pictorial essay with a multimodality approach. J Anat. 2010; 217(6): 633-45. PMID: 21108526. PMC ID: PMC3039176. DOI: 10.1111/j.14697580.2010.01302x.

[52] Mason LW, Dodds A, Makwana N. Tibiofibular Synostosis following Syndesmosis Fixation: A case report. The Foot and Ankle Online Journal. 2010; 3 (3): 3 . DOI: 10.3827/ faoj. 2010.0303.0003.

[53] Botchu R, Douis H, Davies AM, James SL, Puls F, Grimer R. Post-traumatic heterotopic ossification of distal tibiofibular syndesmosis mimicking a surface osteosarcoma. Clin Radiol. 2013; 68(12): e676-9. PMID: 24034551. DOI: 10.1016/j.crad.2013.07.020.

[54] Hinds RM, Lazaro LE, Burket JC, Lorich DG. Risk factors for posttraumatic synostosis and outcomes following operative treatment of ankle fractures. Foot Ankle Int. 2014; 35(2): 141-7. PMID: 24165573. DOI: $10.1177 / 107110$ 0713510913.

[55] Sureka J, Jakkani RK, Ahmed M, Panwar S, Shanker S. Congenital distal tibiofibular synostosis. Radiol Case Rep. 2015; 7(2): 555. PMID: 27326275. PMCID: PMC4899874. DOI: $10.2484 /$ rcrv $7 \mathrm{i} 2.555$.

[56] Droog R, Verhage SM, Hoogendoorn JM. Incidence and clinical relevance of tibiofibular synostosis in fractures of the ankle which have been treated surgically. Bone Joint J. 2015; 97(7): 945-9. PMID: 26130350. DOI: 10.1302/ 0301-620X.97B7.34460.

[57] Marvan J, Dzupa V, Krbec M, SkalaRosenbaum J, Bartoska R, Kachlik D, Baca V. Distal tibiofibular synostosis after surgically resolved ankle fractures: An epidemiological, 
clinical and morphological evaluation of a patient sample. Injury. 2016; 47(11): 2570-2574. PMID: 27645618. DOI: 10.1016/j.injury. 2016.09.007.

[58] Jung ST, Wang SI, Moon YJ, Mubarak SJ, Kim JR. Posttraumatic Tibiofibular Synostosis After Treatment of Distal Tibiofibular Fractures in Children. J Pediatr Orthop. 2017; 37(8): 532536. PMID: 26650579. DOI: 10.1097/BPO.000 0000000000708 .
[59] Sferopoulos NK, Kotakidou R, Petropoulos AS. Myositis ossificans in children: a review. Eur $\mathbf{J}$ Orthop Surg Traumatol. 2017; 27(4): 491-502. PMID: 28275867. DOI: 10.1007/s00590-0171932-x.

[60] Yuen CP, Lui TH. Distal Tibiofibular Syndesmosis: Anatomy, Biomechanics, Injury and Management. Open Orthop J. 2017; 11: 670677. PMID: 29081864. PMCID: PMC5 633698. DOI: $10.2174 / 1874325001711010670$.

Citation: N. K. Sferopoulos. Tibiofibular Synostosis. ARC Journal of Orthopedics. 2018; 3(1): 5-10. doi:dx.doi.org/ 10.20431/2456-0588.0301002.

Copyright: (C) 2018 Authors. This is an open-access article distributed under the terms of the Creative Commons Attribution License, which permits unrestricted use, distribution, and reproduction in any medium, provided the original author and source are credited. 